\title{
Paclitaxel/carboplatin versus topotecan/paclitaxel/carboplatin in patients with FIGO suboptimally resected stage III-IV epithelial ovarian cancer a multicenter, randomized study
}

\author{
Giorgio Bolis ${ }^{a, *}$, Giovanna Scarfone ${ }^{a}$, Francesco Raspagliesi ${ }^{b}$, Giorgia Mangili ${ }^{c}$, \\ Saverio Danese ${ }^{d}$, Paolo Scollo ${ }^{e}$, Domenica Lo Russo ${ }^{f}$, Antonella Villa ${ }^{a}$, \\ Paola Daniela Aimone ${ }^{g}$, Giovanni Scambia ${ }^{f}$ \\ a Second Obstetric and Gynecologic Clinic, University of Milan and Fondazione IRCCS Ca' Granda Ospedale Maggiore Policlinico, Milan, Italy \\ ${ }^{\mathrm{b}}$ Istituto Nazionale Tumori, Milano, Italy \\ c Gynecological Department, University Vita - Salute San Raffaele, Milan, Italy \\ d Divisione di Oncologia, Ospedale S. Anna, Torino, Italy \\ e Ospedale Canizzaro, Catania, Italy \\ ${ }^{\mathrm{f}}$ Ospedale Gemelli, Università Cattolica, Roma, Italy \\ g Clinical Development, Glaxo Smith Kline Pharmaceuticals, Verona, Italy
}

\section{A R T I C L E I N F O}

\section{Article history:}

Received 29 April 2010

Received in revised form 17 June

2010

Accepted 22 June 2010

Available online 29 July 2010

\section{Keywords:}

Ovarian cancer treatment

Paclitaxel

Topotecan

Carboplatin

Survival

\begin{abstract}
A B S T R A C T
Objective: The objective of this prospective randomized phase III trial was to compare paclitaxel plus carboplatin (PC) versus topotecan plus carboplatin and paclitaxel (TPC) in women with suboptimal stage III (residual tumour $>1 \mathrm{~cm}$ ) or stage IV ovarian cancer to evaluate the survival rate and toxicities.

Methods: Eligible for the study were patients aged at least 18 years old with histological/ cytological diagnosis of FIGO stages III (residual tumour $\geqslant 1 \mathrm{~cm}$ after primary surgery) IV epithelial ovarian cancer. Patients were randomized to iv PC on day 1, every 21 days or iv topotecan daily for three days and PC on day 3, every 21 days.

Results: The intention to treat population was made of 326 patients in total, 170 in the PC group and 156 in the TPC group. The life table estimates of survival probabilities at one, three and five years were, respectively, 0.94 (95\% CI: 0.88-0.97), 0.53 (95\% CI: 0.44-0.62) and 0.32 (95\%CI: $0.23-0.42$ ) in the PC group, and 0.92 (95\% CI: $0.86-0.95), 0.52$ (95\% CI: $0.42-0.61$ ), and $0.32(95 \% \mathrm{CI}: 0.22-0.43$ ) in the TPC group (log-rank test at 5 years: ns). The results of the survival analysis based on Cox regression model showed no statistically significant differences between groups ( $p$-value: ns). The number of subjects with at least one event with possible relationship to study medication was $151(88.8 \%)$ in the PC group and 139 (89.1\%) in the TPC group ( $p=\mathrm{ns})$.

Results: In the PC group, 79 patients (23.6\%) experienced at least one Adverse Event (AE) graded as severe and 16 patients (4.8\%) at least one life-threatening AE, whilst in the TPC
\end{abstract}

* Corresponding author: Address: Clinica Ostetrico Ginecologica, Università degli Studi di Milano, via Commenda 12, 20122 Milano, Italy. Tel.: +390 250320262; fax: +390 250320260 .

E-mail address: Giorgio.Bolis@unimi.it (G. Bolis).

0959-8049/\$ - see front matter (C) 2010 Published by Elsevier Ltd.

doi:10.1016/j.ejca.2010.06.124 
group, the number of patients who presented at least one severe or life-threatening AE was $86(24 \%)$ and $37(10.3 \%)$, respectively.

Conclusion: The results of the present study show that the addition of topotecan to a standard paclitaxel/carboplatin regimen in the treatment of advanced epithelial ovarian cancer did not result in significant advantages in terms of survival rate. A slightly worse toxicity profile for TPC was observed.

(c) 2010 Published by Elsevier Ltd.

\section{Introduction}

The therapy for advanced ovarian carcinoma is maximal surgical cytoreduction followed by chemotherapy.

Several trials showed that paclitaxel-carboplatin (PC) combination is as effective as paclitaxel and cisplatin ${ }^{1-3}$ with a better safety profile.

Regimens including a carboplatin plus paclitaxel have become the preferred first-line therapy.

Despite the progress that has been achieved over the years, survival rates in patients with advanced ovarian cancer are still disappointing. ${ }^{4}$

Thus topotecan, gemcitabine, and anthracyclines have been included into first-line regimens for advanced ovarian cancer. $^{5-10}$

According to previous experience of a salvage treatment including carboplatin on day 3 and topotecan as a 3-day administration, ${ }^{5}$ on 2000 started a prospective randomized phase III trial to compare PC versus topotecan plus carboplatin and paclitaxel (TPC) in women with suboptimal stage III (residual tumour $>1 \mathrm{~cm}$ ) or stage IV ovarian cancer to evaluate the survival rate and toxicities. ${ }^{11}$ In this paper we report the final results.

\section{Methods}

This is an open-label, multicentre, randomized study designed to evaluate the efficacy and the toxicities of the association PC versus TPC in patients with FIGO stage III (residual tumour $>1 \mathrm{~cm}$ ) - IV epithelial ovarian cancer.

Eligible for the study were patients aged at least 18 years old with histological/cytological diagnosis of FIGO stages III (residual tumour $\geqslant 1 \mathrm{~cm}$ after primary surgery)-IV epithelial ovarian cancer; performance Status $\leqslant 2$ (ECOG scale); a life expectancy of at least 3 months; presence of at least one indicator lesion to be used for assessment of response (preferably surgery (laparoscopy or laparotomy); no prior chemotherapy; laboratory values: $\mathrm{WBC} \geqslant 3.5 \times 10^{3} \mu \mathrm{l}$, haemoglobin $\geqslant 9.0 \mathrm{~g} / \mathrm{dl}$, neutrophils $\geqslant 1.5 \times 10^{3} \mu \mathrm{l}, \quad$ platelets $\geqslant 100 \times 10^{3} \mu \mathrm{l}$, creatinine $\leqslant 1.5$ $\mathrm{mg} / \mathrm{dl}$, or creatinine clearance $\geqslant 60 \mathrm{ml} / \mathrm{min}$, serum bilirubin $\leqslant$ $2.0 \mathrm{mg} / \mathrm{dl}$, SGOT, SGPT, and alkaline phosphatase $\leqslant 2$ times the upper limit of normal; no noteworthy ECG abnormalities.

Exclusion criteria were concomitant malignancies or previous malignancies within the last five years (excepting basal or squamous cell carcinoma of the skin and carcinoma in situ of the cervix); CNS and/or leptomeningeal metastases; concurrent severe medical problems unrelated to the malignancy which would significantly limit full compliance with the study; history of cardiac diseases, other concurrent chemo- therapy, immunotherapy, radiotherapy, or any other investigational medication for the treatment of the tumour; prior treatment with other chemotherapy regimen.

Eligible patients were randomized by phone by the coordinating centre to receive either iv PC on day 1, every 21 days or iv daily topotecan for three days and PC on day 3, every 21 days.

Study participants were stratified according to FIGO stage of disease (stage III versus stage IV versus carcinosis) and participating centre.

The randomization started in February 2000 and ended in December 2003.

\subsection{Group PC}

Paclitaxel $175 \mathrm{mg} / \mathrm{m}^{2}$ was administered as a 3-h infusion followed by carboplatin AUC 5 given as a 30-min infusion on day 1 every 21 days for 6 cycles. Carboplatin dosage was calculated according to the Cockroft and Gault formula.

\subsection{Group TPC}

Topotecan $1.0 \mathrm{mg} / \mathrm{m}^{2}$ was administered intravenously over $30 \mathrm{~min}$ for three days (day 1-3). Paclitaxel at a dose of $175 \mathrm{mg} /$ $\mathrm{m}^{2}$ given as a 3-h infusion followed by carboplatin AUC 5 given as a 30-min infusion were administered on day 3 every 21 days for 6 cycles. On day 3, the patients got the 3 drugs.

Topotecan dose was not reduced (unless the toxicity was believed to be related to topotecan. In this case, the protocol foresaw to reduce the dose to $0.8 \mathrm{mg} / \mathrm{m}^{2}$ ). Carboplatin and paclitaxel were cut to AUC 4 and $150 \mathrm{mg} / \mathrm{m}^{2}$, respectively, if the platelet count was $<75 \times 10^{3} \mu$ l or the granulocyte count was $<1.0 \times 10^{3} \mu \mathrm{l}$ for $>7$ days at nadir, despite G-CSF therapy in the latter case.

Chemotherapy administration started within 5 weeks since surgery.

During the 6 cycles' period, patients with progressive disease $(\mathrm{PD})$ suspended the study treatment as soon as the progression was detected.

Standard premedication included: clorfenamine maleatum $10 \mathrm{mg}$ im $1 \mathrm{~h}$ before starting paclitaxel, cymetidin $300 \mathrm{mg}$ iv, and hydrocortisone sodium succinate $500 \mathrm{mg}$ iv $30 \mathrm{~min}$ before starting paclitaxel.

Centres were free to perform interval debulking surgery in responding patients in which primary surgery was explorative, laparoscopy, or laparotomy.

Second look surgery was allowed in responding patients with negative CA 125 after 6 cycles of chemotherapy. During second look surgery it was possible to remove residual tumour in patients with partial response (PR). 
Indicators for response evaluation were second look surgery or indicator lesion. These lesions had to meet the criteria for measurable or valuable disease and had to be defined by a tumour imaging assessment (including CT or MRI scan, ultrasound, or chest X-ray), or physical examination. The same diagnostic imaging method was used throughout the study to evaluate the lesions.

Additional chemotherapy, including maintenance or consolidation was not allowed till the progression of the disease.

For patients who did not progress on study and completed the treatment, the investigator conducted the post-treatment assessments every 3 months during the first year, every 4 months during the second year, and every 6 months during the third and the fourth year.

The study protocol did not provide any indication for second line treatment of patients. However, the general policy of participating centres included second-line chemotherapy with a platinum-based compound in case of late recurrence or progression of the disease (i.e. $>12$ months after first-line treatment) and a treatment including anthracyclines in case of early recurrence/progression.

Follow up was updated on 2007.

The study was conducted according to Good Clinical Practice guidelines and the Declaration of Helsinki and received the approval by the Review Boards of the participating centres.

Patients had given their written informed consensus to the study.

\subsection{Statistical consideration and data analysis}

The computation of the sample size considered the main endpoint: survival. The purpose of this study was to compare the rate of overall survival at 3 years in patients receiving the combination of PC versus TPC.

The targeted sample size for this protocol was 350 patients.

Considering an overall survival rate in the PC group of $20 \%$ at 3 years from first diagnosis, this sample size was foreseen to be able to identify an increase in survival rate in the TPC group to $35 \%$ with $80 \%$ of power and alfa 0.05 .

In consideration of the higher survival rate observed at 3 years in the PC group (about $40 \%$ ), we have computed a post hoc computation of the power of the study: we are able to identify a difference in survival rate to $55 \%$ in the TPC group with $80 \%$ of power and alfa 0.05 .

\subsection{Data analysis}

In consideration of the availability of follow up data at the moment of the preparation of the report of the study, 5-years survival rates are presented in this paper for the total population and 4-years survival rates for the analysis in strata of selected variables.

The primary efficacy variable was overall survival.

The secondary efficacy variables were progression free survival and response rate.

The progression free survival was defined as the time interval between randomization to the first documented sign of progression.
Complete response (CR) was defined as (per WHO criteria) complete disappearance of all known measurable and evaluable diseases for a period of at least four weeks. Partial response (PR) was defined as $50 \%$ or greater decrease in the sum of the products of the greatest length and perpendicular width of the largest measurement of all measurable lesions for at least four weeks with no simultaneous increase in a known lesion ( $>25 \%$ ) or appearance of new lesions or increase in valuable disease during this period. Progression was defined as greater than $25 \%$ increase in the sum of the products of the measurable disease, reappearance of measurable disease, clear worsening of valuable disease, appearance of any new lesions, or significant worsening of conditions presumed to be related to malignancy.

Toxicity was recorded according to the WHO recommendations.

For efficacy analysis we considered all randomized patients who received at least one dose of study medication (intention to treat population, ITT).

The per protocol population (PP) included all the evaluable subjects according to the complete criteria defined in the study protocol.

For safety analysis we considered the ITT population.

Kaplan-Meier survival estimates were plotted and the survival probabilities in the two treatment groups were compared using the log-rank test. An additional survival analysis based on Cox regression was planned. The model included terms for performance status: 0-1 versus 2; FIGO staging: III versus IV; histotype: serous versus non-serous; FIGO grading: 1 versus 2/3; residual tumour: $<2 \mathrm{~cm}$ versus $2-5 \mathrm{~cm}$ versus $>5 \mathrm{~cm}$ and/or peritoneal carcinosis; age: $\leqslant 50$ years versus $>50$ years and centre as covariates.

The percentages of patients who showed CR, PR, SD, and $\mathrm{PD}$ were summarised by treatment. The response rates in the two treatment groups were compared by means of the chi-square test or, where appropriate, by the Fisher's exact test.

The number of patients experiencing adverse events and the total number of adverse events (AE) that occurred during the study were calculated. Differences in the number of patients with at least one $\mathrm{AE}$ between the two treatment groups were tested using the chi-square test.

A summary of observed AE has been tabulated according to common toxicity grade (NCI CTC).

The following laboratory data were analyzed by summarising the CTC grade distribution for each visit and the worst CTC grade reported during the study: neutrophils, platelets, haemoglobin, and leucocytes.

The number of patients who underwent supportive therapy (transfusions or G-CSF) was presented for each treatment group.

\section{Results}

A total of 330 patients were enrolled in the study in 28 centres in Italy.

Four patients did not present any evidence of assumption of the study drugs. These patients were excluded from the ITT 
population, represented by 326 patients in total, 170 in the PC group, and 156 in the TPC group.

The PP population included 257 patients in total, 133 in the PC group, and 124 in the TPC group (Fig. 1).

The distribution of study subjects according to selected characteristics and study group is shown in Table 1.

The study groups were similar with regard to age, histotype, stage, grade, residual tumour, and lymph nodal status distribution.

Since the first diagnosis, 166 patients $(97.6 \%)$ in the PC group and 148 patients (94.9\%) in the TPC group underwent surgical procedures for ovarian cancer.

In both treatment groups the most common surgical procedure was laparotomy, reported by 144 patients $(86.7 \%$ of the subjects who had a surgical procedure) in the PC group and by 134 (90.5\%) in the TPC group.

\subsection{Overall survival and progression free survival}

The results of the survival analysis are shown in Fig. 1. The life table estimates of survival probabilities at one, two and three, four and five years were, respectively, 0.94 (95\% CI: $0.88-0.97$ ), 0.71 (95\% CI: 0.63-0.78), 0.53 (95\% CI: 0.44-0.62), 0.41 (95\% CI: $0.32-0.50)$, and 0.32 (95\% CI: $0.23-0.42)$ in the PC group, and 0.92 (95\% CI: 0.86-0.95), 0.77 (95\% CI: 0.69-0.84), 0.52 (95\% CI: $0.42-0.61$ ), 0.41 (95\% CI: $0.31-0.51$ ), and 0.32 (95\% CI: 0.22-0.43) respectively, in the PCT group.

The comparison between the two treatment groups was not statistically significant (log-rank test at 5 years: ns).

Analyses in strata of residual tumour, histotype, and grading showed no statistical difference on survival between both groups, however patients with residual tumour of $1-2 \mathrm{~cm}$ treated with the triple schedule tended to show a higher 4year survival rate $(0.48$ versus 0.66$)$ and subjects with carcinosis a lower one ( 0.53 versus $0.47 p$ ns) (Table 2 ).

The results of the survival analysis based on Cox regression model showed no statistically significant differences between groups ( $p$-value: ns): the relative risk for TPC compared to PC was 0.85 (95\% CI: 0.56-1.29).

The results in the PP population were consistent with those observed in the ITT analysis: the comparison between the two groups was not statistically significant (log-rank test: ns) (data not shown).

The results of the progression free survival analysis are summarised in Figs. $2 \mathrm{a}$ and $2 \mathrm{~b}$. The comparison between the two treatment groups was not statistically significant (log-rank test $p$-value: ns). This result was confirmed for the PP population (log-rank test $p$-value: ns, data not shown).

Data on objective response was available in for $137 \mathrm{pa}$ tients in the PC and 126 in the TPC group. The results of response rates are shown in Table 3.

No significant differences were observed between the two treatment groups in the rate of complete or partial response between the groups ( $p$-value: 0.62 for $C R$ and 0.67 for PR). The results in the PP population were consistent with those observed in the ITT analysis (data not shown).

\subsection{Safety}

The number of subjects with at least one event with possible relationship to study medication was 151 (88.8\%) in the PC group and 139 (89.1\%) in the TPC group ( $p=n s)$.

In the PC group, 79 patients (23.6\%) experienced at least one $\mathrm{AE}$ of severity graded as severe and 16 patients $(4.8 \%)$ at

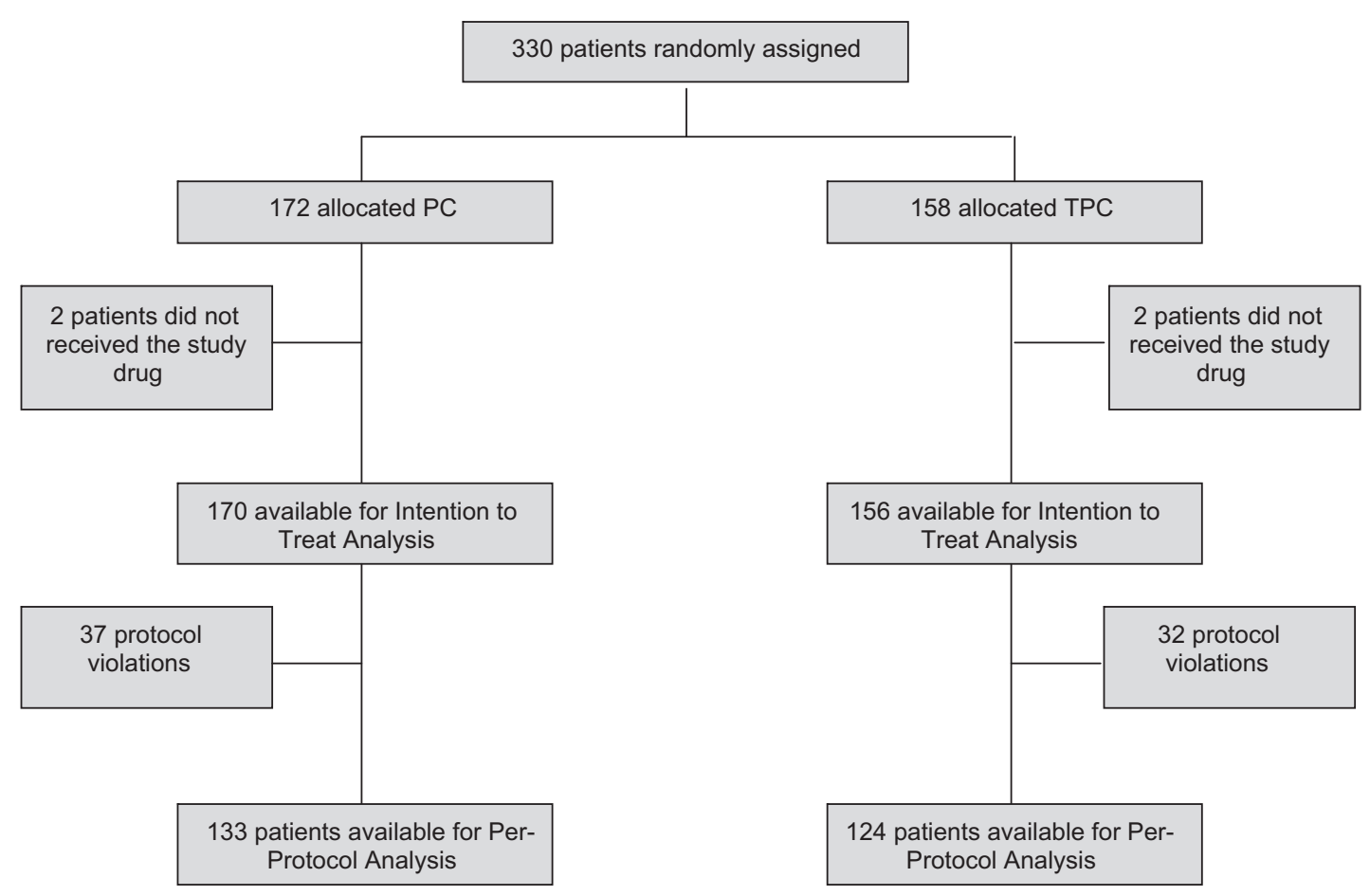

CONSORT trial flow diagram for patients accrued into the trial.

Fig. 1 - CONSORT trial flow diagram for patients accrued into the trial. 


\section{Table 1 - Characteristics of study patients.}

PC group (No. $=170)$

TPC group (No. $=156)$

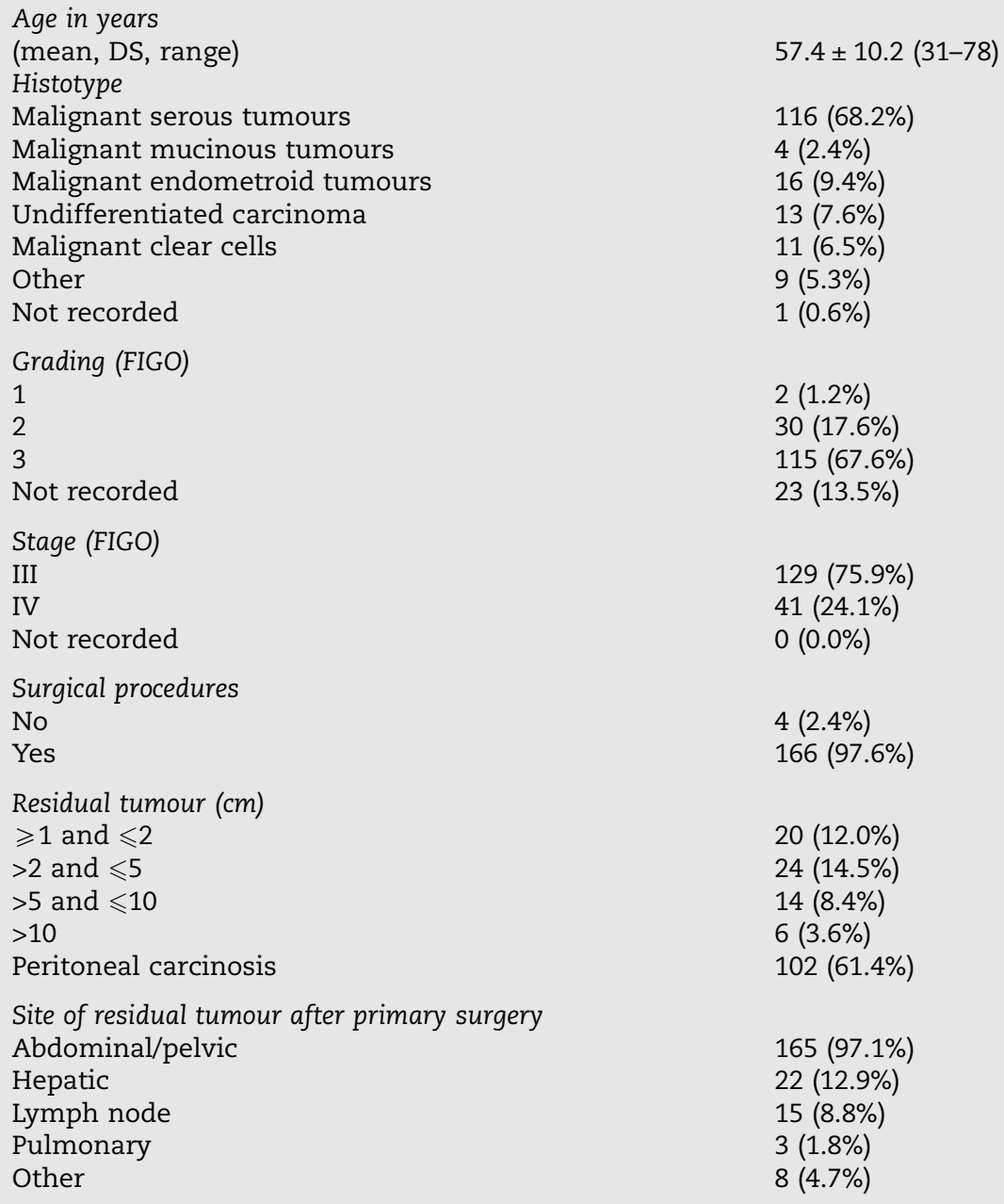

$58.7 \pm 9.4(38-75)$

$117(75.0 \%)$

$0(0.0 \%)$

$3(1.9 \%)$

19 (12.2\%)

$8(5.1 \%)$

$9(5.8 \%)$

$0(0.0 \%)$

$0(0.0 \%)$

$28(17.9 \%)$

$108(69.2 \%)$

$20(12.8 \%)$

123 (78.8\%)

$32(20.5 \%)$

1 (0.6\%)

$8(5.1 \%)$

$148(94.9 \%)$

$19(12.8 \%)$

$22(14.9 \%)$

$11(7.4 \%)$

$5(3.4 \%)$

$91(61.5 \%)$

$150(96.2 \%)$

$15(9.6 \%)$

$29(18.6 \%)$

$6(3.8 \%)$

8 (5.1\%)

Table 2 - Four year overall survival according to study group in the total population and in strata of selected characteristics

\begin{tabular}{lccc} 
& PC group & TPC group & P value \\
\cline { 2 - 4 } & \% Survival $(95 \%$ confidence interval) & \% Survival (95\% confidence interval) \\
\hline Total population & $41(32-50)$ & $41(31-51)$ & \\
Residual tumour $(\mathrm{cm})$ & & & \\
$\geqslant 1$ to $\leqslant 2$ & $48(24-99)$ & $66(36-85)$ & n.s. \\
$>2$ & $57(40-70)$ & $57(37-72)$ & n.s. \\
Carcinosis & $53(41-64)$ & $47(34-64)$ & n..s. \\
Histotype & & & \\
Serous tumour & $50(39-60)$ & $36(49-66)$ & n.s. \\
Other & $61(44-74)$ & & \\
Grading & & $58(33-77)$ & n.s. \\
$1-2$ & $53(31-71)$ & $52(40-63)$ & n.s. \\
3 & $55(44-64)$ & & \\
\hline n.s.: not significant. & & & \\
\hline
\end{tabular}

least one life-threatening AE, whilst in the TPC group, the number of patients who presented at least one severe or life-threatening AE was 86 (24\%) and 37 (10.3\%), respectively.
The most frequent $\mathrm{AE}$ are reported in Table 4.

Fatigue, anaemia, neuthopenia were significantly more frequent in the TPC group. 


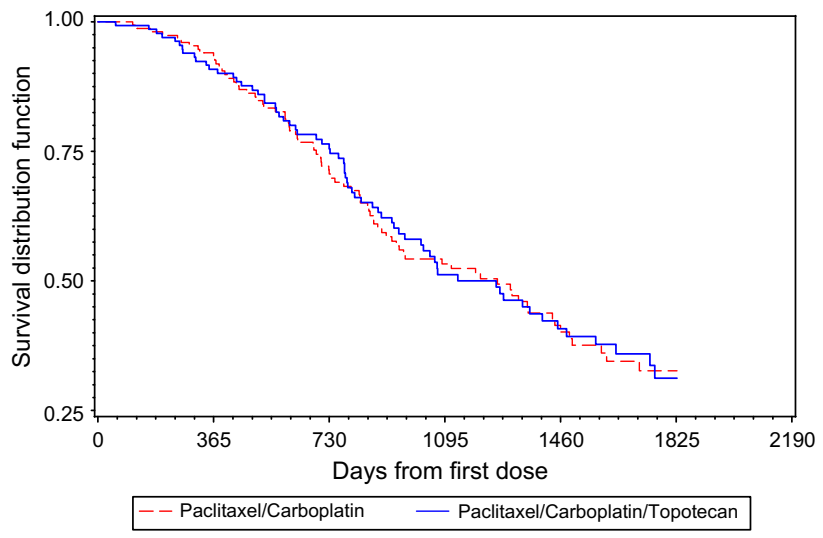

$\begin{array}{lrr}\begin{array}{l}\text { Time interval } \\ \text { (days) }\end{array} & \begin{array}{r}\text { N. of pts } \\ \text { with event }\end{array} & \begin{array}{r}\text { N. of pts } \\ \text { censored }\end{array}\end{array}$

Paclitaxel/Carboplatin

$\begin{array}{lrl}0-365 & 10 & 27 \\ 365-730 & 31 & 11 \\ 730-1095 & 21 & 12 \\ 1095-1460 & 12 & 14 \\ 1460-1825 & 5 & 15 \\ 1825 \quad 2190 & 0 & 12\end{array}$

Fig. 2a - Kaplan-Meier survival plot: time to death.

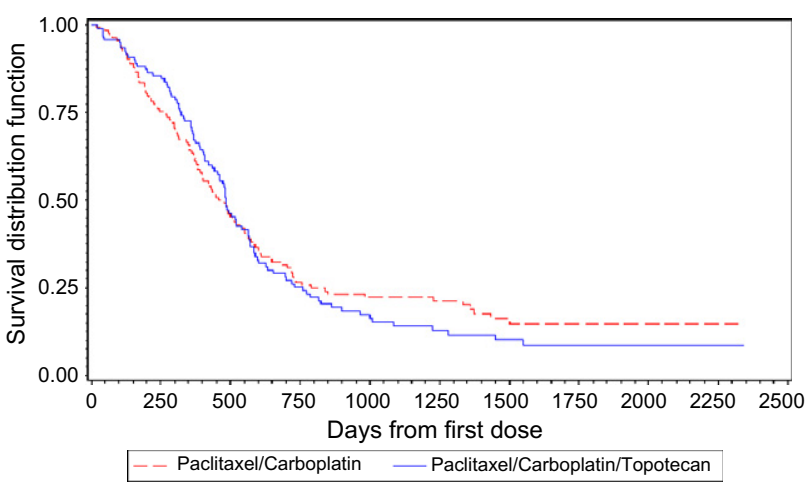

Paclitaxel/Carboplatin/Topotecan

$\begin{array}{lcr}0-365 & 12 & 28 \\ 365-730 & 17 & 15 \\ 730-1095 & 25 & 15 \\ 1095-1460 & 8 & 9 \\ 1460-1825 & 5 & 10 \\ 1825-2190 & 0 & 12\end{array}$

Treatment comparison: paclitaxel/carboplatin vs paclitaxel/carboplatin/topotecan Logrank test Value 0.013 , P-value 0.910

Fig. $2 b$ - Kaplan-Meier survival plot on time to progression.

\subsection{Treatment modification}

The median duration of chemotherapy was 112 days (range 22-198) in the PC group and 115 days (range 24-192) in the TPC group ( $p$ : ns).

The number of patients with at least one course delay was $78(49.1 \%)$ in the PC group and $76(53.1 \%)$ in the TPC group ( $p$ : ns).

The number of patients with at least one paclitaxel dose reduction was 18 (11.3\%) in the PC group and 40 (28.0\%) in

\begin{tabular}{|c|c|c|c|}
\hline & $\begin{array}{l}\text { PC group } \\
\text { No. (\%) }\end{array}$ & $\begin{array}{c}\text { TPC group } \\
\text { No. (\%) }\end{array}$ & $P$ value \\
\hline Complete response & 67 (48.9\%) & $66(52.0 \%)$ & \\
\hline Partial response & 43 (31.4\%) & 43 (33.9\%) & \\
\hline Stable disease & 16 (11.7\%) & $6(4.7 \%)$ & \\
\hline Progressive disease & $11(8.0 \%)$ & $11(8.7 \%)$ & n.s. \\
\hline
\end{tabular}

the TPC group ( $p:<0.001)$ and those with at least one carboplatin dose reduction was respectively 16 (10.1\%) and 44 $(30.8 \%)(p:<0.001)$.

Two patients (1.4\%) at cycle 2 and $1(0.8 \%)$ at cycle 3 required topotecan dose reduction.

The number of patients who completed six treatment cycles was 139 (82.2\%) in the PC group and 121 (77.6\%) in the TPC group (p: 0.29).

\subsection{Supportive therapy}

The number of patients who received transfusions was 16 (11.6\%) in the PC group and $32(26.9 \%)$ in the TPC group ( $p$ : $0.002)$.

The number of patients who received G-CSF at the end of treatment was $10(7.2 \%)$ in the PC group and $30(25.2 \%)$ in the TPC group $(p:<0.001)$.

\section{Discussion}

The primary objective of this study was to evaluate survival in the two following treatment regimens: PC versus TPC in patients with sub optimally-resected stage III or IV ovarian cancer.

The general results show that there were no statistically significant differences between the two treatment regimens in survival rate. Moreover, the comparison between both groups in terms of time to progression and response rate did not show any statistically significant difference.

With regard to safety and tolerability, the rate of drug-related adverse events was similar in the two groups, whilst the risk of drug-related serious adverse events was higher in the group of patients receiving additional topotecan.

The risk of anaemia and leucopenia was higher in the TPC than in the PC group and the percentage of patients requiring transfusions or supportive G-CSF therapy was lower in the PC group than in the TPC group.

The results of this study should be discussed in comparison with published data.

Several randomized clinical trials have tested the role of triple cytotoxic therapy for advanced ovarian cancer in comparison with standard treatment with carboplatin or cisplatin plus paclitaxel. Triple schedules included anthracycline, gemcitabine, and topotecan. In general no differences emerged. ${ }^{12}$

In particular, some studies randomized clinical trials and one phase II study have included topotecan in the triple schedule. 
Table 4 - Summary of patients with adverse events by common toxicity grade.

\begin{tabular}{|c|c|c|}
\hline & $\begin{array}{l}\text { Paclitaxel/ } \\
\text { carboplatin } \\
(N=170)\end{array}$ & $\begin{array}{c}\text { Paclitaxel/ } \\
\text { carboplatin/topotecan } \\
(n=156)\end{array}$ \\
\hline \multicolumn{3}{|l|}{ Allergy } \\
\hline Mild & 8 (4.71\%) & 8 (5.13\%) \\
\hline Moderate & $1(0.59 \%)$ & $4(2.56 \%)$ \\
\hline Severe & $1(0.59 \%)$ & $5(3.21 \%)$ \\
\hline Life threatening & $1(0.59 \%)$ & $0(0.00 \%)$ \\
\hline Not indicated & $0(0.00 \%)$ & $1(0.64 \%)$ \\
\hline$P$ value & ns & \\
\hline \multicolumn{3}{|l|}{ Anorexia } \\
\hline Mild & $2(1.18 \%)$ & $1(0.64 \%)$ \\
\hline Moderate & $1(0.59 \%)$ & $0(0.00 \%)$ \\
\hline$P$ value & n.s. & \\
\hline \multicolumn{3}{|l|}{ Arthralgia } \\
\hline Mild & $4(2.35 \%)$ & $5(3.21 \%)$ \\
\hline Moderate & $4(2.35 \%)$ & $2(1.28 \%)$ \\
\hline$P$ value & n.s. & \\
\hline \multicolumn{3}{|l|}{ Fatigue } \\
\hline Mild & $2(1.18 \%)$ & $8(5.13 \%)$ \\
\hline Moderate & $2(1.18 \%)$ & $0(0.00 \%)$ \\
\hline Severe & $0(0.00 \%)$ & $1(0.64 \%)$ \\
\hline Not indicated & $1(0.59 \%)$ & $0(0.00 \%)$ \\
\hline$P$ value & 0.05 & \\
\hline \multicolumn{3}{|l|}{ Febrile neutropenia } \\
\hline Mild & $0(0.00 \%)$ & $1(0.64 \%)$ \\
\hline Life threatening & $0(0.00 \%)$ & $1(0.64 \%)$ \\
\hline$P$ value & n.s. & \\
\hline \multicolumn{3}{|l|}{ Nausea } \\
\hline Mild & $28(16.47 \%)$ & 31 (19.87\%) \\
\hline Moderate & $16(9.41 \%)$ & $17(10.90 \%)$ \\
\hline Severe & $1(0.59 \%)$ & $3(1.92 \%)$ \\
\hline$P$ value & n.s. & \\
\hline \multicolumn{3}{|l|}{ Neurotoxicity } \\
\hline Mild & 51 (30.00\%) & 47 (30.13\%) \\
\hline Moderate & $6(3.53 \%)$ & $3(1.92 \%)$ \\
\hline Severe & $2(1.18 \%)$ & $2(1.28 \%)$ \\
\hline Not indicated & $0(0.00 \%)$ & $1(0.64 \%)$ \\
\hline$P$ value & n.s. & \\
\hline \multicolumn{3}{|l|}{ Mucositis } \\
\hline Mild & $0(0.00 \%)$ & $2(1.28 \%)$ \\
\hline Moderate & $1(0.59 \%)$ & $1(0.64 \%)$ \\
\hline$P$ value & n.s. & \\
\hline \multicolumn{3}{|l|}{ Vomiting } \\
\hline Mild & 7 (4.12\%) & $9(5.77 \%)$ \\
\hline Moderate & $8(4.71 \%)$ & $16(10.26 \%)$ \\
\hline Severe & $1(0.59 \%)$ & $3(1.92 \%)$ \\
\hline Life threatening & $0(0.00 \%)$ & $1(0.64 \%)$ \\
\hline$P$ value & n.s. & \\
\hline \multicolumn{3}{|l|}{ Thrombosis } \\
\hline Severe & $0(0.00 \%)$ & $1(0.64 \%)$ \\
\hline$P$ value & n.s. & \\
\hline \multicolumn{3}{|l|}{ Haemoglobin } \\
\hline $0(>10 \mathrm{~g} / \mathrm{dL})$ & $102(60.0 \%)$ & $50(32.1 \%)$ \\
\hline $1(=10 \mathrm{~g} / \mathrm{dL})$ & $1(0.6 \%)$ & $3(1.9 \%)$ \\
\hline $2(\geqslant 8 e<10 \mathrm{~g} / \mathrm{dL})$ & $64(37.6 \%)$ & $93(59.6 \%)$ \\
\hline $3(\geqslant 6.5<8 \mathrm{~g} / \mathrm{dL})$ & $2(1.2 \%)$ & $10(6.4 \%)$ \\
\hline $4(<6.5 \mathrm{~g} / \mathrm{dL})$ & $1(0.6 \%)$ & $0(0.0 \%)$ \\
\hline$P$ value & $<0.01$ & \\
\hline
\end{tabular}

\section{Table 4 (continued)}

Paclitaxel/ Paclitaxel/ carboplatin carboplatin/topotecan $(N=170) \quad(n=156)$

$\begin{array}{lll}\text { Leucocytes } & & \\ 0\left(\geqslant 3.5 \times 10^{3} \mathrm{ul}\right) & 71(41.8 \%) & 51(32.7 \%) \\ 1\left(\geqslant 3 \mathrm{e}<3.5 \times 10^{3} \mathrm{ul}\right) & 39(22.9 \%) & 27(17.3 \%) \\ 2\left(\geqslant 2 \mathrm{e}<3 \times 10^{3} \mathrm{ul}\right) & 56(32.9 \%) & 61(39.1 \%) \\ 3\left(\geqslant 1 \mathrm{e}<2 \times 10^{3} \mathrm{ul}\right) & 4(2.4 \%) & 17(10.9 \%) \\ P \text { value } & 0.004 & \\ \text { Neutrophils } & & \\ 0\left(\geqslant 2 \times 10^{3} \mathrm{ul}\right) & 24(14.1 \%) & 29(18.6 \%) \\ 1\left(\geqslant 1.5 \mathrm{e}<2 \times 10^{3} \mathrm{ul}\right) & 35(20.6 \%) & 23(14.7 \%) \\ 2\left(\geqslant 1 \mathrm{e}<1.5 \times 10^{3} \mathrm{ul}\right) & 71(41.8 \%) & 42(26.9 \%) \\ 3\left(\geqslant 0.5 \mathrm{e}<1 \times 10^{3} \mathrm{ul}\right) & 31(18.2 \%) & 52(33.3 \%) \\ 4\left(<0.5 \times 10^{3} \mathrm{ul}\right) & 9(5.3 \%) & 10(6.4 \%) \\ P \text { value } & 0.004 & \end{array}$

Note: only the event with the maximum toxicity grade was considered for each patient and $\mathrm{AE}$.

In the analysis of the Gynaecologic Cancer InterGroup study, the patients who received PC plus topotecan reported a 3-weeks decrement in median progression free survival in comparison with patients receiving standard CP treatment. ${ }^{10}$

Pfisterer et al. ${ }^{9}$ (2006) conducted a randomized trial including 1308 patients with stage IIB-IV ovarian cancer. These patients were randomized to receive six cycles of paclitaxel and carboplatin followed by either four cycles of topotecan or surveillance on a 3-week per cycle schedule. The median survival was 43.1 months for the topotecan group and 44.5 months for the surveillance one.

Further, in a small phase II study 343 patients with advanced ovarian cancer and $>1 \mathrm{~cm}$ residual disease were treated with sequential carpoblatin (AUC 5 days 1 and 22) paclitaxel (1.75 mg m(-2) days 43 and 64) and topotecan $1.5 \mathrm{mg} \mathrm{m}(-2)$ daily for 5 days. The best overall response was $77 \%$ and the median survival was 22.2 months. ${ }^{13}$

The standard dose of topotecan as monotherapy in first or second line therapy for ovarian cancer is $1.5 \mathrm{mg} / \mathrm{m}^{2}$ over 5 days. In the Gynaecologic Cancer InterGroup study, topotecan was given at in the dose of $1.25 \mathrm{mg} / \mathrm{m}^{2}$. In the present series topotecan was given at the dose of $1.0 \mathrm{mg} / \mathrm{m}^{2}$ over 3 days and the effective given dose was lower.

Delivery of the third drug in an adequate dosage is difficult. $^{12}$ Further, concomitant delivery of topotecan, paclitaxel with a platinum compound has been shown feasible with cisplatin, ${ }^{14}$ but not with carboplatin.

Earlier phase I studies including topotecan and cisplatin suggested that myelosuppression was lower if cisplatin was given on day 5 rather than day $1 .^{12}$

In this study carboplatin was given on day 3 .

It has been suggested that more aggressive therapy may have greater impact in patients with small volume residual disease. Along this line, in the Gynaecologic Cancer InterGroup analysis, the TPC schedule showed a, not statistically significant, better survival rate than PC one in patients with microscopic residual tumour, but not with residual tumour $\leqslant 1 \mathrm{~cm}$ or $>1 \mathrm{~cm}$. ${ }^{10}$ 
We have analyzed overall survival rates in the strata of residual tumour, histotype and grading. No statistical difference emerged, but patients with residual tumour of $1-2 \mathrm{~cm}$ treated with the triple schedules had a higher, not significant, 4-year survival rate $(0.66$ versus 0.48 ).

In this analysis also progression free survival rates were similar in the two groups. In this study second look surgery was allowed. It has been shown that second surgical assessment of small-volume disease may change the determination of time to progression, ${ }^{15}$ but in this randomized trial second look surgery was performed in both the groups in a similar frequency.

In conclusion, the results of the present study show that the addition of topotecan to a standard paclitaxel/carboplatin regimen in the treatment of advanced epithelial ovarian cancer did not result in significant advantages in terms of survival rate, time to progression and response. These findings are in general agreement with the result of the large data set of the Gynaecologic Cancer InterGroup study. A slightly worse toxicity profile for TPC as well as a more frequent need for more G-CSF was observed.

\section{Role of funding source}

This study was funded by SmithKline Beecham and GlaxoSmithKline.

\section{Conflict of interest statement}

None declared.

\section{Acknowledgement}

The authors wish to thank Fabio Parazzini for his help in the discussion of the data and in the preparation of the manuscript.

\section{R E F E R E N C E S}

1. Neijt JP, Engelholm SA, Tuxen MK, et al. Exploratory phase III study of paclitaxel and cisplatin versus paclitaxel and carboplatin in advanced ovarian cancer. J Clin Oncol 2000;18:3084-92.

2. du Bois A, Luck HJ, Meier W, et al. A randomized clinical trial of cisplatin/paclitaxel versus carboplatin/paclitaxel as first-line treatment of ovarian cancer. J Natl Cancer Inst 2003;95:1320-9.

3. Ozols RF, Bundy BN, Greer BE, et al. Phase III trial of carboplatin and paclitaxel compared with cisplatin and paclitaxel in patients with optimally resected stage III ovarian cancer: a Gynecologic Oncology Group study. J Clin Oncol 2003;21:3194-200.

4. Piccart MJ, Bertelsen K, Stuart G, et al. Long-term follow-up confirms a survival advantage of the paclitaxel-cisplatin regimen over the cyclophosphamide-cisplatin combination in advanced ovarian cancer. Int $J$ Gynecol Cancer 2003;13(Suppl. 2):144-8.

5. Bolis G, Scarfone G, Villa A, Parazzini F. Phase II trial of topotecan, carboplatin, and paclitaxel as front-line therapy in suboptimal advanced epithelial ovarian cancer. Gynecol Oncol 2001;81:331-3.

6. Du Bois A, Pfisterer J. Future options for first-line therapy of advanced ovarian cancer. Int J Gynecol Cancer 2005;15(Suppl. 1): 42-50.

7. du Bois A, Weber B, Rochon J, et al. Addition of epirubicin as a third drug to carboplatin-paclitaxel in first-line treatment of advanced ovarian cancer: a prospectively randomized gynecologic cancer intergroup trial by the Arbeitsgemeinschaft Gynaekologische Onkologie Ovarian Cancer Study Group and the Groupe d'Investigateurs Nationaux pour l'Etude des Cancers Ovariens. J Clin Oncol 2006;24:1127-35.

8. Rose PG, Greer BE, Horowitz IR, Markman M, Fusco N. Paclitaxel, carboplatin and pegylated liposomal doxorubicin in ovarian and peritoneal carcinoma: a phase I study of the Gynecologic Oncology Group. Gynecol Oncol 2007;104:114-9.

9. Pfisterer J, Weber B, Reuss A, et al. Randomized phase III trial of topotecan following carboplatin and paclitaxel in first-line treatment of advanced ovarian cancer: a gynecologic cancer intergroup trial of the AGO-OVAR and GINECO. J Natl Cancer Inst 2006;98:1036-45.

10. Bookman MA, Brady MF, McGuire WP, et al. Evaluation of new platinum-based treatment regimens in advanced-stage ovarian cancer: a Phase III Trial of the Gynecologic Cancer Intergroup. J Clin Oncol 2009;27:1419-25.

11. Scarfone G, Scambia G, Raspagliesi F, et al. A multicenter randomized phase III study comparing paclitaxel/caroplatin (PC) versus topotecan/paclitaxel/carboplatin (TPC) in patients with stage III (residual tumour $>1$ after primary surgery) and IV ovarian cancer (OC). J Clin Oncol 2006;24(Suppl. 18s):5003.

12. Hoskins PJ. Triple cytotoxic therapy for advanced ovarian cancer: a failed application, not a failed strategy. J Clin Oncol 2009;27:1355-8.

13. Guppy AE, Nelstrop AE, Foster T, et al. A phase II study of sequential carboplatin, paclitaxel and topotecan in patients with previously untreated advanced ovarian cancer. $\mathrm{Br} \mathrm{J}$ Cancer 2004;90:810-4.

14. Armstrong DK, Bookman MA, McGuire W, Bristow RE, Schilder JM. A phase I study of paclitaxel, topotecan, cisplatin, and filgrastim in patients with newly diagnosed advanced ovarian epithelial malignancies: a Gynecologic Oncology Group study. Gynecol Oncol 2007;105:667-71.

15. Greer BE, Bundy BN, Ozols RF, et al. Implications of secondlook laparotomy in the context of optimally resected stage III ovarian cancer: a non-randomized comparison using an explanatory analysis: a Gynecologic Oncology Group study. Gynecol Oncol 2005;99:71-9. 